\title{
Effect of amber powder on endometrial ultrastructure and MAPK pathway in endometriosis model rats
}

\author{
Xiaona $\mathrm{Ma}^{1}$, Yanhui $\mathrm{Wu}^{1}$, Bingbing $\mathrm{Li}^{2}$, Zheng Wang ${ }^{1}$, Xiujuan Zhu ${ }^{1}$, Jing Jia ${ }^{1}$, \\ Yanfeng Liư ${ }^{2 \star}$ \\ ${ }^{1}$ Third Affliated Hospital of Beijing University of Chinese Medicine, ${ }^{2}$ Dongzhimen Hospital, Beijing University of Chinese \\ Medicine, Beijing, China
}

*For correspondence: Email: yangfengliu1970@yahoo.com; Tel: 0086-75643208236

\begin{abstract}
Purpose: To explore the therapeutic role of amber powder in endometriosis by investigating its effect on endometrial ultrastructure, ERK1/2, P38MAPK, and NF-KB mRNA pathways and C$S R C / E F R / E R K 1 / 2$ proteins.

Methods: Sprague Dawley (SD) rats were randomly divided into blank group, disease model group (untreated), amber powder high-dose group, amber powder medium-dose group, amber powder lowdose group and danazol group. Morphological changes in endometrial cells were studied using transmission electron microscopy. The expression of ERK1/2, p38MAPK, and NF-KB mRNA in endometrial tissues of each group was determined using quantitative real-time polymerase chain reaction (qRT-PCR). Immunohistochemistry was utilized for the measurement of C-SRC/EFR/ERK1/2 pathway protein expression.

Results: The endometriosis rats treated with a high-, medium- and low-dose amber powder showed a decrease in the volume of ectopic lesions, compared with the untreated disease model group. The expressions of ERK1/2, p38MAPK, NF-KB mRNA, and C-SRC/EFR/ERK1/2 protein were higher in the eutopic and ectopic endometrial tissues in untreated disease group than those in normal control group. Moreover, treatment of endometriosis rats with amber powder revealed a reduction in the expressions of ERK1/2, p38MAPK, NF-KB mRNA and C-SRC/EFR/ERK1/2 proteins in eutopic and ectopic endometrium tissues.

Conclusion: Amber powder reduces ectopic lesions and slows down the development of endometriosis, probably via inhibition of MAPK pathway genes in eutopic and ectopic endometrial tissues.
\end{abstract}

Keywords: Amber powder, Endometriosis, Ultrastructure, MAPK pathway

\begin{abstract}
This is an Open Access article that uses a fund-ing model which does not charge readers or their institutions for access and distributed under the terms of the Creative Commons Attribution License (http://creativecommons.org/licenses/by/4.0) and the Budapest Open Access Initiative (http://www.budapestopenaccessinitiative.org/read), which permit unrestricted use, distribution, and reproduction in any medium, provided the original work is properly credited.

Tropical Journal of Pharmaceutical Research is indexed by Science Citation Index (SciSearch), Scopus, International Pharmaceutical Abstract, Chemical Abstracts, Embase, Index Copernicus, EBSCO, African Index Medicus, JournalSeek, Journal Citation Reports/Science Edition, Directory of Open Access Journals (DOAJ), African Journal Online, Bioline International, Open-J-Gate and Pharmacy Abstracts
\end{abstract}

\section{INTRODUCTION}

Endometriosis (EMs) refers to abnormal growth of endometrial tissue outside the uterine and generally involves pelvis lining, ovaries and fallopian tubes [1]. The incidence of this disease in women of childbearing age is $10-15 \%$. It is a multifactorial condition that causes various gynecological problems including dysmenorrhea, infertility and chronic pelvic pain in women. The 
pathogenesis of EMs involves the adhesion and proliferation of endometrial tissues, invasion of endometrial cells, and neoangiogenesis.

The process of EMs development comprises the coordination of various signal transduction pathways, such as mitogen-activated protein kinase (MAPK) signaling pathway which are associated with different immune processes, inflammatory response, apoptosis, cell proliferation, and differentiation [3-4]. Previous studies have shown that amber powder has a regulatory effect on EMs rats with immune imbalance [4].

Endometriosis can be treated using various western and traditional medicines. Amber powder is a traditionally used Chinese medicine for endometriosis. This medicine is a unique powder that is acquired from the fossilized resin of Pinus succinifera. Amber powder nourishes and promotes blood circulation [5].

The aim of the present study was to assess the influence of amber powder on the ultrastructure of eutopic and ectopic endometrium in EMs rats, MAPK pathway ERK1/2, p38MAPK, NF-KB mRNA, and C-SRC/EFR/ERK1/2 protein expression.

\section{EXPERIMENTAL}

\section{Animals}

Ninety female SD rats of specific pathogen-free (SPF) grade, 3 months age, and $180-220 \mathrm{~g}$ weight were used in this study. The rats were kept in animal laboratory of Beijing University of Chinese Medicine with temperature control at 20 - $25{ }^{\circ} \mathrm{C}$, the relative humidity of $40-60 \%$, and cleanliness level II (i.e., a dust-free area). The experimental protocol of this study was reviewed, approved (approval no. BUCM/12032017/33-01) by the Animal Ethics Committee of Beijing University of Chinese Medicine and executed according to international guidelines for animal use in laboratory experiments [5].

\section{Drugs}

Amber powder is composed of various plants including Rehmannia, wild celery (Angelica), radix astragali (Chinese name Wuwu), scripus rhizome (Chinese name Sanling), and Toxicodendron vernicifluum (Chinese name Qishu). Chinese medicine granules were produced from Kangrentang Pharmaceuticals, Beijing, China and were gifted by a Chinese Pharmacy. Danazol capsules (0.2 g/granule, manufactured by Jiangsu Lianhuan
Pharmaceutical, Batch number: 20110102) were donated by a Western Pharmacy. Reverse transcription cDNA kit (US promega, A5001), fluorescent quantitative PCR kit (US promega, A6002), Trizol (US invitrogen, 15596018), immunohistochemical SABC kit, and Nakasu Jinqiao SP-9001 were purchased through a market source.

\section{Model establishment and grouping}

Rats were nourished for one week with ad libitum access to rat chow and tap water, randomly numbered, and labeled by staining. The rats were distributed into six groups, i.e. blank group, disease model group, high dose amber powder group, medium-dose amber powder group, low dose amber powder group, and danazol group using random number table method so that each group contained 10 rats. EMs model was established using the international common classical model-autologous abdominal wall transplantation method [4]. To induce EMs, diethylstilbestrol was administered at a dose of $0.02 \mathrm{mg} / \mathrm{kg}$ for 5 consecutive days [4].

\section{Drug administration}

The drugs were administered one week after the model establishment. Normal saline was given to the blank group and the disease model group. Rats of various groups received drugs as stated here: low dose amber group $12.5 \mathrm{~g}$ of amber powder $/ \mathrm{kg}$ of rat, medium-dose amber group 25 $\mathrm{g} / \mathrm{kg}$, high dose amber group $50 \mathrm{~g} / \mathrm{kg}$, and danazol group $0.0625 \mathrm{~g} / \mathrm{kg}$.

\section{Indicators and methods}

Four weeks after the operation, the rats were anesthetized and then sacrificed by decapitation. Laparotomy technique was used to observe transplant growth in rats. The pathological changes of the transplanted tissues were observed under a light microscope. The epithelial cells or interstitial cells with endometrial tissue were seen in the transplant tissues taken as successful modeling standard. The rats showing successful RMs modeling exhibited bloody vesicles containing clear liquid and cystic solids (Figure 1). The eutopic endometrial tissue was taken from the blank group, on the other hand, both ectopic and eutopic endometrial tissues were taken from other groups. The collected tissues were fixed in either $2 \%$ paraformaldehyde, $2.5 \%$ glutaraldehyde precooled at $4{ }^{\circ} \mathrm{C}$, or $4 \%$ formaldehyde solution, followed by the processing of electron microscope specimens, qRT- PCR specimens, and paraffin sections, respectively. 


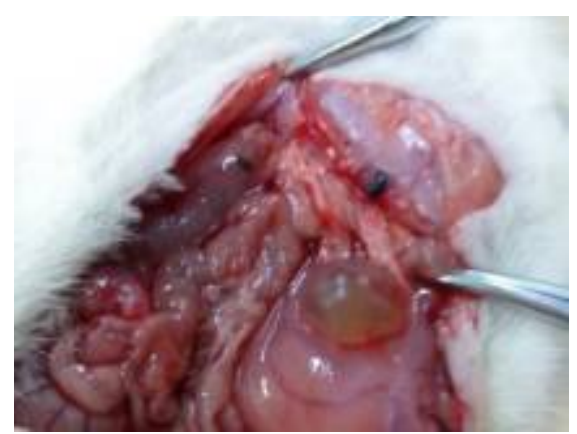

Figure 1: Successful modeling of EMs showing clear vesicles and angiogenesis on the surface.

\section{Preparation of electron microscope specimens}

The tissue was taken out after $2 \mathrm{~h}$ fixation and cut into $1 \mathrm{~mm}^{3}$ slice. After washing with buffer for 20 min, acetone-gradient dehydration was performed and soaked by Epon 618 epoxy resin embedding machine, Beijing. The tissue was then embedded in a porous rubber embedding template, placed in an oven for drying, and thin sections were prepared. Ultra-thin sections (50$70 \mathrm{~nm}$ ) were prepared after positioning, doublestained, and then observed by using transmission electron microscopy (Hitachi, model $\mathrm{H}$ - Observed under 7650).

Determination of ERK1/2, p38MAPK and NFKB mRNA expression by qRT-PCR

\section{Primer design and synthesis}

The primers (Table 1) of each index were synthesized with Bioengineering Biotechnology (Shanghai) Co., Ltd.

\section{Extraction of $\mathrm{mRNA}$}

Tissue mRNA was extracted by Trizol one-step extraction method, and the purity of the RNA sample was assessed by spectrophotometer. After reverse transcription of mRNA, the obtained cDNA was heated to approximately $93^{\circ} \mathrm{C}$ on the AB17500 machine, and the double-stranded template DNA was broken by hydrogen bonds in effects of heat, forming single-stranded DNA, and denaturation of the template DNA was completed; complete renaturation was achieved by combining the primers with template DNA in a system having $55^{\circ} \mathrm{C}$; the temperature was increased to about $72{ }^{\circ} \mathrm{C}$. Under the action of Taq enzyme, dNTP was utilized as the raw material, leading to the synthesis of a DNA strand complementary to the template, resulting in the extension of the primer. Finally, the obtained data were analyzed numerically.

\section{Immunohistochemical study of C- SRC/EFR/ERK1/2 protein expression}

In the blank group, the eutopic endometrial tissue was taken, while both eutopic and ectopic endometrial tissue were taken in other groups and fixed in $4 \%$ formaldehyde solution for the preparation of paraffin sections. Paraffin sections were preheated at $60{ }^{\circ} \mathrm{C}$, routinely dewaxed into water, and autoclaved for antigenic repair for 10 minutes, then cooled to room temperature. The sections were then washed three times with PBS for $5 \mathrm{~min}, 0.3 \% \mathrm{H}_{2} \mathrm{O}_{2} \mathrm{RT}$ for $10 \mathrm{~min}$ (closed endogenous HRP), PBS for $5 \mathrm{~min}$ again, and $10 \%$ normal goat serum for 10 min (block tissue endogenous IgG, Fc-R). Primary antibody working solution was added, incubated, and washed on day 2 with PBS for 5 min. Then, secondary antibody working solution was added, incubated, and washed three times with PBS for $5 \mathrm{~min}$ again, and observed under microscope. Afterward, the degree of color development was controlled by washing using running water and counterstained with hematoxylin, followed by differentiation using $1 \%$ hydrochloric acid alcohol. It was wash thoroughly to turn blue, transparent, neutral gum seal. Immunohistochemical sections were studied by professional image analysis system Image-Pro Plus6.0, and the mean optical density values $(M O D=I O D /$ area, where IOD is an integrated optical density) was used as the evaluation parameters of C-SRC, EFR, and ERK1/2 protein expression intensity.

Table 1: PCR primer sequence

\begin{tabular}{llcc}
\hline Gene & $\begin{array}{l}\text { Oligonu- } \\
\text { cleotide }\end{array}$ & Sequence & $\begin{array}{c}\text { PCR product } \\
\text { size (bp) }\end{array}$ \\
\hline ERK1/2 & Forward & CGATGAAAACCTGAACACCA & 324 \\
& Reverse & ATCAACCTTCCCCCAAAATC & 324 \\
P38mapk & Forward & ATCATTCACGCCAAAAGGAC & 298 \\
& Reverse & CTTCATTCACAGCGAGGTTG & 298 \\
$N F-k B$ & Forward & GAACTTGTGGGGAAGGACTG & 301 \\
& Reverse & GGGCACGGTTATCAAAAATC & 301 \\
\hline
\end{tabular}


Three fields were randomly observed for each slice, and the mean value was taken as the observation result of each slice.

\section{Statistical analysis}

Statistical calculations were conducted using Statistical Package for the Social Sciences (SPSS) version 20.0 software. $P \leq 0.05$ was set as the statistically significant level. Comparison between two groups was by t-test or MannWhitney $U$ test. Multiple groups $(\geq 3)$ were analyzed by one-way ANOVA or by KruskalWallis $\mathrm{H}$ test.

\section{RESULTS}

\section{Ectopic lesion size}

The morphology of the ectopic lesions was observed and the volume (in $\mathrm{mm}^{3}$ ) of the grafts was measured with a vernier caliper by determining its length, width, and height. According to results, the volume of the ectopic lesions in the disease model group was larger than all other groups. After treating with amber and danazol, the volume of the ectopic lesions was significantly $(p<0.01)$ reduced. There were non-significant $(p>0.05)$ differences among the amber high, middle and low dose groups and the danazol group (Figure 2).

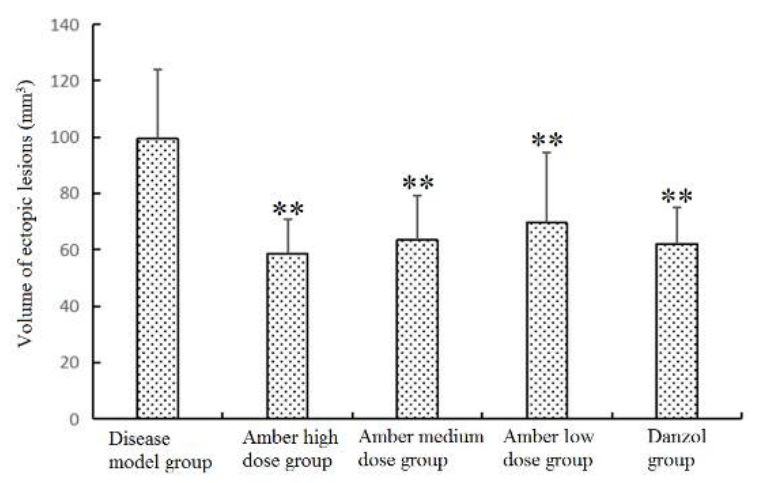

Figure 2: The size of ectopic lesions in each group: After treatment with amber powder and danzol, the volume of ectopic lesions is reduced as compared to the disease model group ${ }^{* *}$ indicates that $p<0.01$ )

\section{Tissue/cell morphology}

The normal endometrial epithelial cells in the blank group were densely arranged and columnar. Cell villous was dense, glandular epithelial cells were normal in morphology; cell connection was clear, the nuclear position was normal, and their mitochondria and rough endoplasmic reticulum were normal. In the disease model group, a large number of mitochondria were observed in the endometrial cells; density of mitochondrial intercondylar matrix was increased; rough endoplasmic reticulum was expanded; nuclear morphology was irregular; perinuclear space was dilated; nuclear membrane was sunken; nucleoplasm ratio was inverted, and nucleolus was slightly enlarged and marginal.

In the disease model group, the ectopic endometrial epithelial cells showed pseudostratification; cell connections were relatively poor; villi were short and sparse; density of mitochondrial inter-matrix was high; rough endoplasmic reticulum was expanded; nuclear morphology was irregular, and some cells were binuclear having sunken nuclear membrane and marginal nucleolus. In the high, medium and low dose of amber powder group, the ectopic endometrial epithelial cell connections were poor; villi were short and sparse; density of mitochondrial intercondylar matrix was high; rough endoplasmic reticulum was slightly expanded; nuclear membrane was sunken, and nucleolus was marginal. In the danazol group, the ectopic endometrial epithelial cells showed pseudo-stratification; microvilli were short; cells were poorly connected; multiple vacuoles were seen; rough endoplasmic reticulum was reduced, and inflammatory cell infiltration was observed in the basal layer (Figure 3).

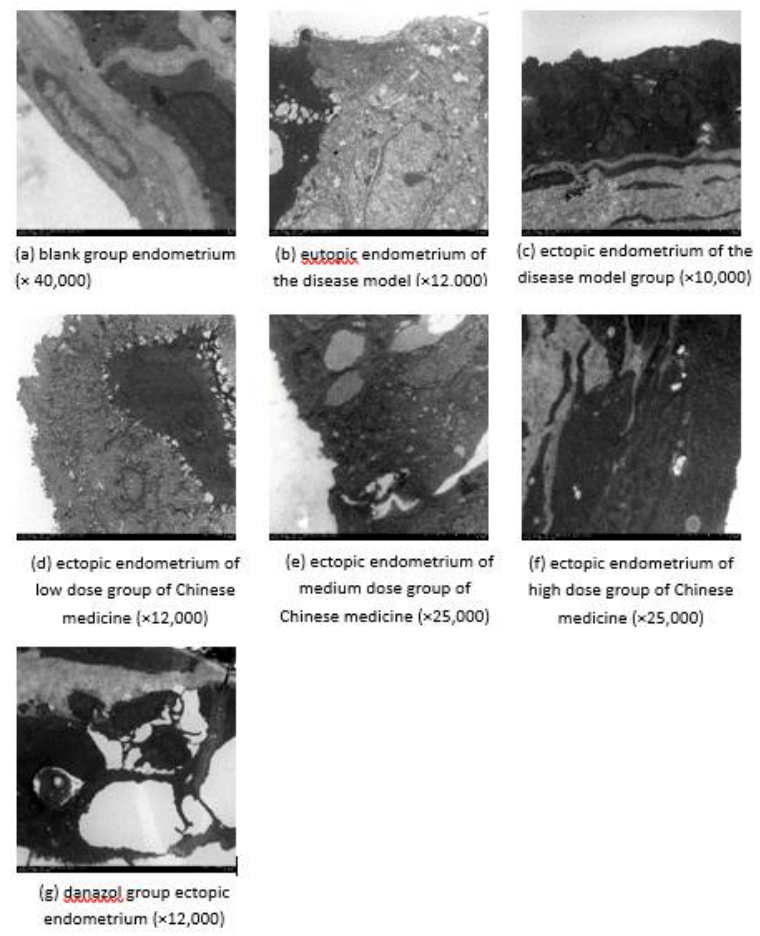

Figure 3: Ultrastructure of normal endometrial rats, eutopic and ectopic endometrium model rats by transmission electron microscopy 
Expressions of ERK1/2, P38MAPK and NF-KB mRNA pathways in the endometrium

The expression of ERK $1 / 2$, p38MAPK, and NFKB mRNA in the eutopic and ectopic endometrial tissues of the disease model group was significantly high $(p<0.01)$ as compared with the blank group. Moreover, the expressions of ERK $1 / 2$ and NF-KB mRNA in the ectopic endometrial tissue of the disease model group were significantly $(p<0.05)$ higher than that of eutopic endometrium. However, there was nonstatistical $(p>0.05)$ difference in the expression of p38MAPK mRNA between the eutopic and ectopic endometrial tissues of the disease model group. In addition, amber treatment significantly $(p<0.01)$ decreased the expressions of ERK $1 / 2$, p38MAPK, and NF-kB mRNA in eutopic and ectopic endometrium of EMs rats (Figure 4).

\section{Expression of endometrial C-SRC/EFR/ERK1/ 2 proteins}

The expression of C-SRC/EFR/ERK1/2 in the eutopic and ectopic endometrial tissues of the disease model group was significantly high $(p<$ 0.01 ) as compared with the blank group.

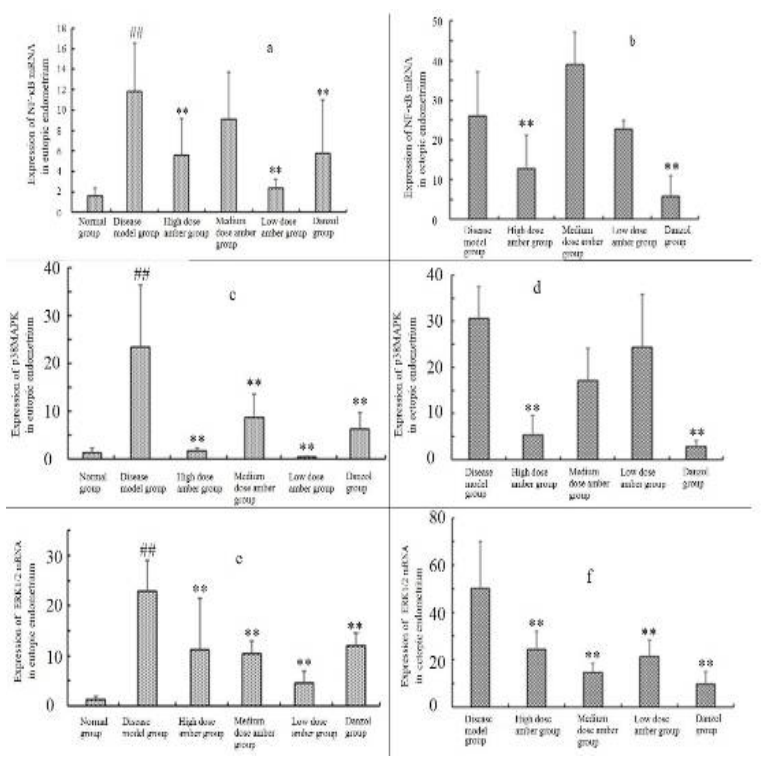

Figure 4: Effect of amber powder on ERK1/2, p38MAPK, and NF-kB mRNA pathways: Amber powder significantly $(p<0.01)$ down-regulates the expression of ERK1/2, p38MAPK, and NF-KB mRNA in eutopic and ectopic endometrial tissues of endometriosis rats, as compared with the disease model group and the blank group. Where \#\# and ** represents $p<0.01$ in comparison with the disease model group and the blank group. All the data are described as mean \pm standard deviation

In addition, the expression of C-SRC/EFR in the ectopic endometrial tissue of the disease model group was significantly $(p<0.05)$ higher than that of eutopic endometrium. However, there was non-statistical $(p>0.05)$ difference in the expression of ERK $1 / 2$ between the eutopic and ectopic endometrial tissues of the disease model group. Furthermore, amber treatment significantly $(p<0.05)$ decreased the expression of C-SRC/EFR/ERK1/2 in eutopic and ectopic endometrium.

\section{DISCUSSION}

In this study, the ultrastructural characteristics of the endometrial cells showed that the eutopic and ectopic endometrial cells in the disease model group were multinuclear, hyperplasic, more mitochondria; rough endoplasmic reticulum expansion, as compared with the normal endometrial cell structure in the blank group. On the other hand, ectopic endometrium cells in the amber-treated groups and the danazol group showed vacuolar degeneration, microvilli deficiency, nuclear malformation, nuclear sputum and various degrees of necrosis and apoptosis. These changes in the ectopic endometrial ultrastructure showed that amber powder may play a therapeutic role in EMs by inhibiting the growth of ectopic lesions at the cellular level. Endometrial invasive implantation of endometriosis is a complex process involving multiple factors, in which MAPK pathway plays an important role [6,5]. The MAPK signaling pathway is activated by hormones and cytokines. Through phosphorylation, it is transferred to the nucleus to regulate expressions of transcription factors and cell activity. Here, it plays a crucial role in cell mitosis, migration, metabolism, apoptosis, and various processes involved in the inflammation and angiogenesis [7]. The development of EMs is closely related to estrogen that mainly exerts its biological effects through ERK1/2 and p38MAPK signaling pathways. It has been reported that ERK $1 / 2$ responds to various growth factors or other external mitogen signals to promote proliferation, regulate differentiation, and enhance the antiapoptotic ability of the cell. Whereas, P38MAPK is activated by inflammatory cytokines and is responsible for the signal transduction of various emergency response cells. It is essential for normal immune and inflammatory responses and is involved in the functional response of macrophages and neutrophils, which are key cells involved in the pathogenesis of EMs [8-10].

This study used autologous abdominal wall transplantation to construct endometriosis model rats. The experimental results showed that endometriosis is common in the ectopic and ectopic endometrium of rats with endometriosis disease. There were high expressions of ERK1/2 
and P38MAPK mRNA, which were consistent with the results of a previous study [11]. The high expression of MAPK signaling pathway in the eutopic endometrium of EMs rats may promote the invasion, proliferation, and growth of the intima, and fit the proposed "eutopic endometrium determinism". It is found that gene expression in the endometrium of EMs patients is different from that of non-EMs patients, thus EMs patients have particular characteristics in many aspects [11]. In the present study, amber powder administered to EMs model rats reduced the expression of ERK1/2 and p38MAPK mRNA in eutopic and ectopic endometrium of EMs rats, and down-regulated the expression of MAPK pathway gene in endometrial tissues. MAPK regulates a variety of cellular transcription factors, such as NF-KB that is a dimeric form of transcription factor family composed of complex polypeptide subunits widely distributed in eukaryotic cells. As a hub of signaling pathways, activated NF-kB participation in the expression of a variety of target genes is a non-specific transcription factor ubiquitous in cells. It is the intersection of multiple signal transcription pathways and participates in the physiological and pathological processes of immune, inflammation, stress and other reactions [11]. The promoters of most cytokine genes associated with endometriosis have functional NF-KB binding sites, thus NF-KB may be involved in the development of EMs by mediating immune-inflammatory responses. Based on a previous study [12], selective inhibition of NF-KB activity is expected to be a new and effective treatment for endometriosis. The results of this study showed that NF-KB is highly expressed in eutopic and ectopic endometrial tissues of endometriosis rats compared with normal endometrial tissue. Conclusively, amber powder reduces the expression of NF-kB mRNA in the eutopic and ectopic endometrium of EMs rats.

\section{CONCLUSION}

MAPK pathway contributes to endometriosis development. Amber powder reduces ectopic lesions and slows down the development of endometriosis. Its mechanism of action may be attributed to the inhibition of MAPK pathway genes in eutopic and ectopic endometrial tissues and decrease in C-SRC/EFR/ERK1/2 protein expression.

\section{DECLARATIONS}

\section{Conflict of interest}

No conflict of interest is associated with this work.

\section{Contribution of authors}

We claim that this study was finished by the writers mentioned in this paper and all the liabilities relating to claims concerning the content of this article will be accepted by the writers. XM, YW, and BL conceived the paper. $\mathrm{XM}, \mathrm{YW}, \mathrm{BL}$, and $\mathrm{ZW}$ drafted the outline and wrote the draft of the article. JJ and $Y L$ reviewed and edited the manuscript. ZW, XZ, JJ, and $Y L$ reviewed and contributed in detail analysis of the lab findings of the patients. $\mathrm{XZ}, \mathrm{JJ}$, and $\mathrm{YL}$ reviewed the manuscript and provided his valuable comments to improve it and contributed towards the finalization of the manuscript.

\section{Open Access}

This is an Open Access article that uses a funding model which does not charge readers or their institutions for access and distributed under the terms of the Creative Commons Attribution License (http://creativecommons.org/licenses/by/ 4.0) and the Budapest Open Access Initiative (http://www.budapestopenaccessinitiative.org/rea d), which permit unrestricted use, distribution, and reproduction in any medium, provided the original work is properly credited.

\section{REFERENCES}

1. Matsuzaki S, Canis $M$, Vaurs-Barrière $C$. DNA microarray analysis of gene expression profiles in deep endometriosis using laser capture microdissection. Mol Hum Reprod 2004; 10(10): 719-728.

2. Yang $Z$, Li C, Wang $X$. Dauricine induces apoptosis, inhibits proliferation and invasion through inhibiting NFkappa $B$ signaling pathway in colon cancer cells. Cell Physiol 2010; 225(1): 266-275.

3. Papa S, Bubici C, Zazzeroni F. The NF-kappa Bmediated control of the JNK cascade in the antagonism of programmed cell death in health and disease. Cell Death Differ 2006; 13 (5): 712-729.

4. Vernon $M$, Wilson $A$. Study on the surgical induction of endometriosis in the rats. Fertil Steril 1985; 44: 782-786.

5. National Institutes of Health Office of Laboratory Animal Welfare (2002). Public Health Service policy on the humane care and use of laboratory animals. Bethesda, MD: NIH. Retrieved September 27, 2011.

6. Kobel M, Pohl G, Schmitt WD. Activation of mitogenactivated protein kinase is required for migration and invasion of placental site trophoblastic tumor. $A m \mathrm{~J}$ Pathol 2010; 167(3): 879-885.

7. Tartaglia M, Gelb BD, Zenker M. Noonan syndrome and clinically related disorders. Best Pract Res Clin Endocrinol Metab 2011; 25(1): 161-179.

8. Yan L, Cao X, Zeng S. Associations of proteins relevant to MAPK signaling pathway (p38MAPK-1, HIF-1 and HO-

Trop J Pharm Res, September 2019; 18(9): 1850 
1) with coronary lesion characteristics and prognosis of peri-menopausal women. Lipids Health Dis 2016; 15(1): 187192.

9. Deng L, Zhang L, Zhao $H$. The role of p38MAPK activation in spinal dorsal horn in remifentanil-induced postoperative hyperalgesia in rats. Neurol Res 2016; 38(10): 929-936.

10. Zhu L, Yuan $C$, Huang $L$. The activation of $p 38 M A P K$ and JNK pathways in bovine herpesvirus 1 infected MDBK cells. Vet Res 2016; 47(1): 91.
11. Defrere S, Gonzalez-Ramos R, Lousse JC, Colette S, Donnez $O$, Donnez J, Van Langendonckt $A$. Insights into iron and nuclear factor-kappa $B$ (NF-kappa B) involvement in chronic inflammatory processes in peritoneal endometriosis. Histol Histopathol 2011; 26(8): 1083.

12. Zheng $Y$, Liu $X$, Guo SW. Therapeutic potential of andrographolide for treating endometriosis. Hum Reprod 2012; 27(5): 1300. 